\title{
Identification and Functional Analysis of LsMNPV Anti-apoptosis Genes
}

\author{
Yu-Sin Kim ${ }^{2, \#}$, Hua-Zhong Xiao ${ }^{1, \#}$, En-Qi Du ${ }^{1}$, Guo-Shuai Cai ${ }^{1}$, Song-Ya Lu ${ }^{1, *}$ and Yi-Peng Qi ${ }^{1, *}$ \\ ${ }^{1}$ State Key Laboratory of Virology, College of Life Sciences, Wuhan University, Wuhan, P. R. China 430072 \\ ${ }^{2}$ College of Life Science, KIM IL SUNG University, Pyongyang, D.P.R.K
}

Received 12 January 2007, Accepted 9 March 2007

\begin{abstract}
Three anti-apoptosis genes, Ls-iap2, iap3 and $p 49$ were found in Leucania separata multiple nuclear polyhedrovirus. Amino acid sequence homology of Ls-IAP2 and Ls-IAP3 with Op-IAP2 and Op-IAP3 from Orgyia pseddotsugata MNPV were $20 \%$ and $42 \%$, while that of Ls-P49 is $28 \%$ with Sl-P49 from Spodoptera littorolis MNPV. Ls-IAP2 contains one baculoviral IAP repeat (BIR) domain followed by a RING domain, while Ls-IAP3 contains two BIRs and a RING. Ls-P49 contains a reactive site loop, predicted cleavage site $\left(K_{K L D}{ }^{74} \downarrow G\right)$ that is different from Sl-P49 (TVID ${ }^{94} \downarrow$ G). Expressed Ls-iap3 or Ls-p49 under presence of actinomycin D in SF9 cells, DNA ladder assay revealed that Ls- IAP3 or Ls-P49 could block the apoptosis of SF9 cells induced by actinomycin D. Replication of p35 deficient-mutant Autographa californica MNPV in SF9 cells was also rescued when Ls-iap3 or Ls-p49 was expressed transiently. No anti-apoptotic activity was observed for LsIAP2. The results showed that both of Ls-IAP3 and Ls-P49 were functional apoptotic suppressors in SF9 cells.
\end{abstract}

Keywords: Anti-apoptosis, LsMNPV, Ls-IAP, Ls-p49

\section{Introduction}

Apoptosis or programmed cell death is a normal physiological cell suicide program that is highly conserved among vertebrates and invertebrates (Du et al., 1999). Apoptosis plays an important role during normal development and tissue homeostasis eliminating unwanted cells, including damaged and virus-infected, from the organism. Apoptosis acts as a host defense mechanism by which virally infected cells are

\#Authors contributed equally to this work.

*To whom correspondence should be addressed.

Tel: 86-27-68754131; Fax: 86-27-68754131

E-mail: Lvsy68@sina.com.cn (S.Y. Lu), qiyipeng@whu.edu.cn (Y.P. Qi) eliminated to limit the propagation of viruses (Pei et al., 2002). To overcome this response, many viruses carry genes whose products inhibit apoptosis (Means et al., 2003).

In baculovirus, two types of anti-apoptotic genes, $p 35$ and inhibitor of apoptosis (iap) have been identified (Clem, 2001). The $p 35$ gene from Autographa californica multiple nucleopolyhedrovirus (AcMNPV) is required to prevent apoptosis during the virus infection of Sf21 cells, a cell line derived from the Lepidopteran insect Spodoptera frugiperda (Kamita et al., 1993; Dai et al., 1999; Huang et al., 2001). P35, a 35$\mathrm{kDa}$ protein encoded by the p35 gene of the AcMNPV, inhibits a broad range of caspases, including human and insect caspases (Pei et al., 2002). The most remarkable feature of P35 structure is the presence of a large loop domain associated with caspase called reactive site loop (RSL), protruding above a central $\beta$-sheet core (Pei et al., 2002).

Recently, p49 was identified from Spodoptera littorolis MNPV (S1-p49) as a second member of the $p 35$ family (Du et al., 1999). Sl-p49 encodes a predicted $49 \mathrm{kDa}$ protein that showed $48.8 \%$ identity to $p 35$ (Pei et al., 2002). P49 is able to inhibit insect and human effector caspases, require cleavage at its $\operatorname{Asp}^{94}\left({ }^{91} \mathrm{TVTD}^{94}\right)$ for the anti-apoptotic function.

The first iap gene was discovered in Cydia pomonella granulovirus (CpGV) and Orgyia pseddotsugata MNPV (OpMNPV), during a genetic screen for genes that could rescue the AcMNPV p35 mutant phenotype (Crood et al., 1997). Cellular homologues of iap gene were widely present in baculovirus, yeast, drosophila, nematode and mammal cells (Huang et al., 2001). Recently, iaps have also been indentified in lepidopteran insects that include Tricoplusia ni, Spodoptera frugiperda and Bombyx mori, Spodoptera litura (Ikeda et al., 2004).

Baculovirus $I A P$ s are currently classified into five members, IAP1-IAP5, based on amino acid sequence homology (Luque et al., 2001). Functional analysis have revealed that only IAP3 is capable of blocking apoptosis in cells infected with Cydia pomonella granulovirus (CpGV) or Orgyia pseudotsugata MNPV (OpMPV) (Means et al., 2003), although these viruses possess three and four IAPs, respectively. It is IAP1 and IAP2 
in Epiphyas postvittana MNPV (EppoMNPV), but not IAP3 and IAP4 that exhibit anti-apoptotic activity (Day et al., 1999).

IAP proteins have distinctive primary structure. They contain one to three copies of baculoviral IAP repeat (BIR) domains and most IAPs also contain a RING domain near their C-termini. The BIR domain contains a highly conserved arrangement of $\mathrm{Cys} / \mathrm{His}$ residues forming a stable fold that chelates zinc [Maguire et al., 2000]. The BIR region was also found to interact with regulators of IAPs including Grim, Reaper, and Hid from Drosophila and may also mediate homo-oligomerization (Seshagiri et al., 1999). The RING finger is a common zinc binding motif that also exists in other cellular proteins. Recent studies showed that several IAPs are inhibitors of caspases and that BIR and RING domains play an important role in apoptosis suppression (Clem and Miller, 1994).

Leucania separata multiple nuclear polyhedrosis virus (LsMNPV) genome was first sequenced by our laboratory in 2002. There are three putative anti-apoptosis genes: Ls-iap2, iap 3 and $p 49$ in the genome of LsMNPV. Here we report the gene cloning, structural and functional study of Ls-iap2, Lsiap 3 and Ls- $p 49$. Our results demonstrate that both Ls-IAP3 and Ls-P49 are functional apoptotic suppressors in SF9 cell, but Ls-IAP2 has no anti-apoptosis function.

\section{Materials and Methods}

Cells, viruses and plasmid. Spodoptera frugiperda pupal ovarian cell line (SF9) cells were cultured at $27^{\circ} \mathrm{C}$ in Graces medium (Gibco/BRL), supplemented with 10\% fetal bovine serum (Gibco/ BRL). $p 35$ deficient-mutant AcMNPV (AcMNPV $\Delta$ p35/pol ${ }^{+}$), pIZ/ V5His are gifted from Dr. Lungang Yao.

Expression vector construction. The primers used to amplify LsIAP2, Ls-IAP3 and Ls-p49 genes from LsMNPV genome were used as follows: Ls-IAP2 F (5'-GGAATTCAGATATGGAGACTC GTGCAAA-3'), Ls-IAP2 R (5'-CGGGTCTAGAAGAAAAATTCG AATCAGAG-3'), Ls-IAP3 F (5'-GGAATTCAAACATGGATTCG CTGGAG), Ls-IAP3 R (5'-CGGTCTAGAAGGAGAATAATAGA CACGAATGAT-3'), Ls-p49 F (5'-CGAATTCTCTCAAGCCTCA CCGCA-3'), Ls-p49 R (5'-CGGGTCTAGATGAATATCAATGTAC AGATTGG-3'). PCR fragments were digested with EcoRI and $X b a \mathrm{I}$ and then inserted into the EcoRI and $X b a \mathrm{I}$ sites in pIZ/V5His to produce pIZ-IAP2, pIZ-IAP3 and pIZ-p49, respectively.

Protein expression and Western-blot. SF9 cells were seeded into 24-well plates at about $60 \%$ confluence and maintained at $27^{\circ} \mathrm{C}$. Transfection was performed with $0.8 \mu \mathrm{g}$ expression plasmid and reporter plasmid using Cellfectin reagent according to the manufacture protocol (vitrogen). Cells were harvested and lysed at $24 \mathrm{~h}, 72 \mathrm{~h}$ after transfection. The expression protein was subjected to SDSPAGE and was evaluated by Western- blot using antibody for $6 \mathrm{His}$ tag or poyhedrin.

DNA extraction. DNA fragments produced with apoptosis were extracted for $2 \mathrm{~h}$ at $37^{\circ} \mathrm{C}$ from cell samples (SF9 cell) with a $10 \mathrm{mM}$ Tris (pH 8.0), $1 \mathrm{mM}$ EDTA-1\% Sodium dodecyl sulfate (SDS) buffer containing $70 \mu \mathrm{g} / \mathrm{ml}$ of protenase $\mathrm{K}$, and $1 \mathrm{M} \mathrm{NaCl}$ (final concentration) was added. The extracts were treated with phenolchloroform and precipitated with ethanol, and resuspended DNA was analyzed by agarose gel electrophoresis.

Flow cytometry. Cell samples were collected by centrifugation at 3,000 rpm for $5 \mathrm{~min}$ and then washed three times using PBS buffer ( $\mathrm{pH}$ 7.0). The cell pellet was resuspended in $1 \mathrm{ml}$ PBS ( $\mathrm{pH} 7.0$ ). RNase A (Gibco) was added in the solution to the final concentration of $50 \mu \mathrm{g} / \mathrm{ml}$ and the cells were incubated at $37^{\circ} \mathrm{C}$ for $30 \mathrm{~min}$. Then PI (Gibco) was added to the solution to the final concentration of $50 \mu \mathrm{g} / \mathrm{ml}$ and incubated at $37^{\circ} \mathrm{C}$ for $30 \mathrm{~min}$. After washing twice cells were then fixed in $70 \%$ ethanol and flow analysis was performed on Bechman-coulter XL (Beckman coulter).

Microscopy observation. Marker rescue assays were performed as described previously (Clem and Miller, 1994). Routinely, $1 \mu \mathrm{g}$ $\mathrm{V} \Delta p 35 / \mathrm{pol}^{+} \mathrm{DNA}$ or $50 \mu \mathrm{g} / \mathrm{ml}$ actinomycin D and $1 \mu \mathrm{g}$ test DNA were cotransfected into $4 \times 10^{5}$ SF9 cells by lipofectin. 3-4 days after transfection, the cells were examined the presence of polyhedra and apoptotic bodies with light microscopy.

Software analysis. Computer- assisted domain structure of Ls-p49 was based on the Predict Protein Server (Rost et al., 2003). Sl-p49 was used model for Ls-p49. Analysis of Ls-IAPs sequences was performed using DNA analysis programs DNAMAN.

\section{Results}

Sequence comparison and structural analysis of Ls-p49 and Ls-IAPs. Ls-p49 is 1,335 bp and encodes an about 49 $\mathrm{kDa}$ protein which contains one putative caspase cleavage sites $\mathrm{KKLD}^{74} \downarrow_{\mathrm{G}}$ and shares $28 \%$ amino acid sequence identity with S1-P49 (Fig. 1). Ls-IAP2 is 831 bp and encodes an about $30 \mathrm{KDa}$ protein. Ls-IAP2 contains a BIR domain at $\mathrm{N}$-terminus and a RING finger motif at C-terminus (Fig. 2A). Ls-IAP2 shares 20, 19, 20, 21 and 19\% amino acid sequence identity with Op-IAP2, Ep-IAP2, Tn-IAP, Sf-IAP, Bm-IAP, respectively. Ls-IAP3 is $744 \mathrm{bp}$ and encodes an about $28 \mathrm{KDa}$ protein. Similar to Op-IAP3, Ls-IAP3 protein contains two BIR domains followed by a RING domain near its C-terminus (Fig. 2B). Ls-IAP3 shares 42\%, 42\%, 31\%, 30\% and 35\% amino acid sequence identity with Op-IAP3, Cp-IAP3, TnIAP, Sf-IAP and Bm-IAP, respectively. The identity between Ls-IAP2 and Ls-IAP3 was 22\%.

Expression of Ls-p49 and Ls-IAPs in SF9 cells. Ls-p49 and Ls-IAPs were amplified using PCR from LsMNPV genome.

PCR fragments were digested with EcoRl and Xbal, and then inserted into $E c o \mathrm{Rl}$ and $X b a \mathrm{l}$ sites of pIZ/V5His to produce pIZ-IAP2, pIZ-IAP3 and pIZ-p49, respectively. pIZ/ V5His plasmid contains the actin promoter of Drosophila, which can initiate gene expression in insect cells. 


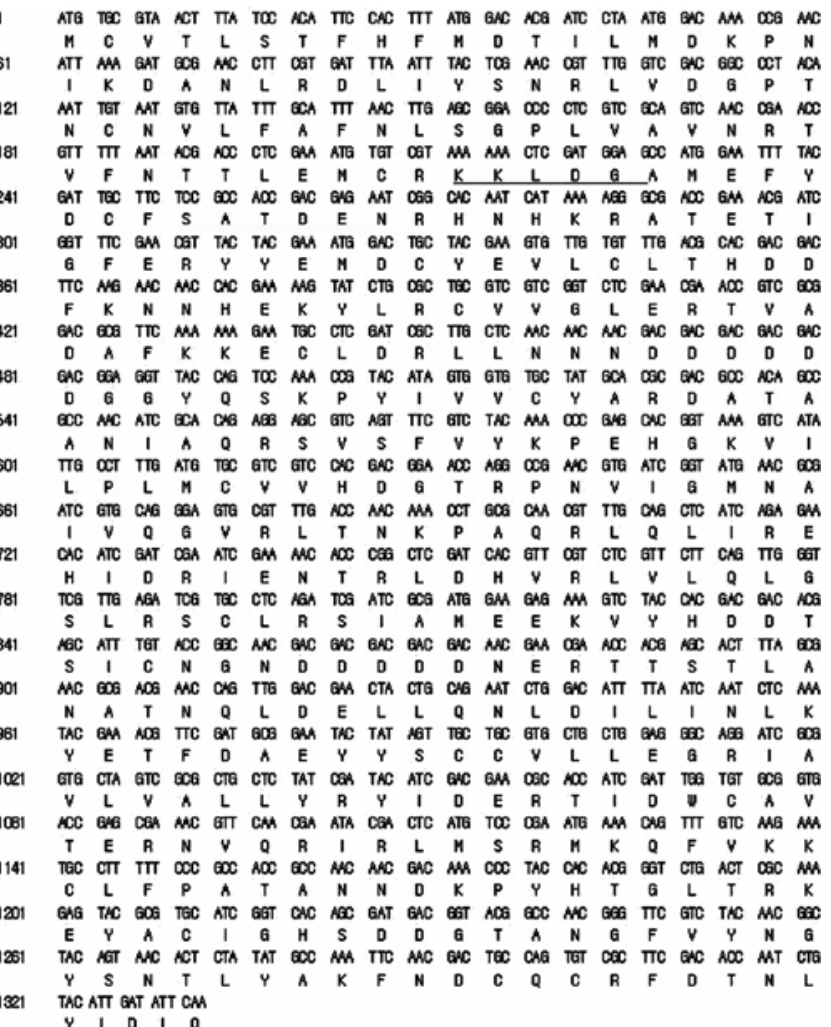

Fig. 1. Nucleotide and predicted amino acid sequence of Ls-p49. The putative caspase cleavage sites are underlined.

$12 \mathrm{~h}$ after transfecting SF9 cells with pIZ-IAP2, pIZ-IAP3 or pIZ-p49, actinomycinD (ActD) was added and incubated for $24 \mathrm{~h}$. Then the expressed Ls-IAP2, Ls-IAP3 or Ls-P49 proteins was subjected to SDS-PAGE analysis and Western blotting. The results showed that Ls-P49 and Ls-IAPs were expressed under the control of actin promoter of Drosophila (Fig. 3A and B).

Ls-P49 and Ls-IAP3 inhibited SF9 cell apoptosis induced by actinomycin $\mathbf{D}$. ActD can block the cell protein synthesis and thus cause the cells to undergo apoptosis. $12 \mathrm{~h}$ prior to the addition of ActD, cells were transfected with pIZ-IAP2, pIZIAP3 or pIZ-p49 and then the transfected cells were treated with ActD for $24 \mathrm{~h}$ to induce apoptosis. After treated with ActD for $24 \mathrm{~h}$, the number of SF9 cells that were mock transfected or transfected with pIZ-IAP2 was reduced and obvious characteristic of apoptosis was observed (Fig. 4B and C). While the cells transfected with pIZ-IAP3 or pIZ-p49 $12 \mathrm{~h}$ prior to the addition of ActD did not show obvious characteristic of apoptosis after $24 \mathrm{~h}$ (Fig. 4D and E).

To examine DNA fragments produced with apoptosis, whole cell DNA was extracted and then subjected to agarose gel electrophoresis. The results showed that the DNA extracted from the cells transfected with pIZ-IAP3 or pIZ-p49 $12 \mathrm{~h}$ prior to addition of ActD was intact (Fig. 5 lane 3 and 4), but the DNA in ActD-treated cells that were mock transfected
A

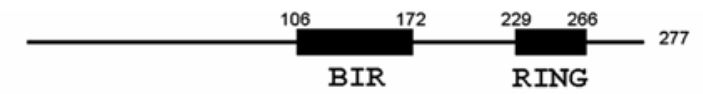

B
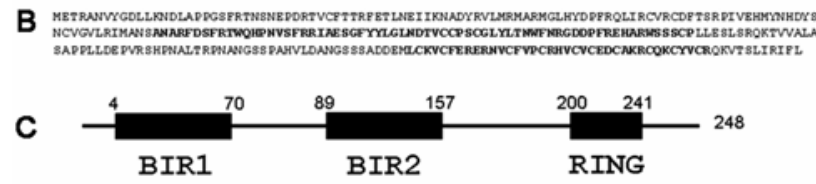

D

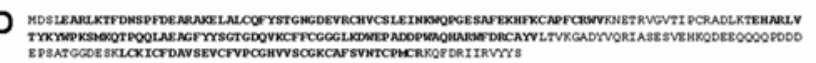

\section{E}

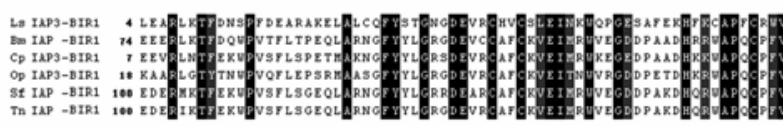

F

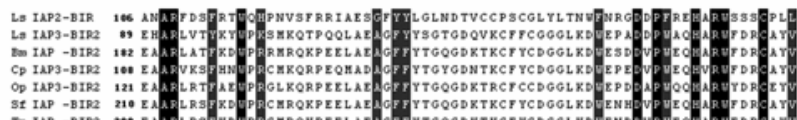

G

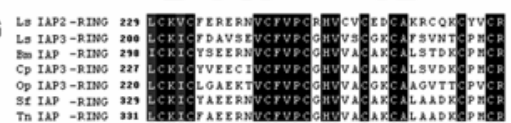

$\mathrm{H}$

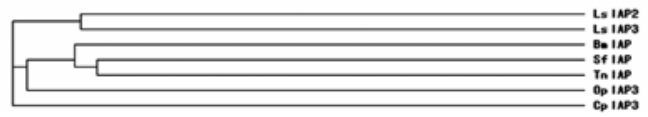

Fig. 2. Comparison of Ls-IAPs amino acid sequences and the predicted domain topology. (A)The BIR and RING domains of Ls-IAP2 are depicted. (B)The predicted amino acid sequence of Ls-IAP2 is presented. (C)The BIR and RING domains of LsIAP3 are depicted. (D)The predicted amino acid Sequence of LsIAP3 is presented. Sequence alignments of the BIR1 (E), BIR2 (F) and RING $(\mathrm{G})$ domains of Ls-IAP2 and Ls-IAP3 with the corresponding domains of other iap family members are shown. Black and gray indicates identical amino acids. $(\mathrm{H})$ Phylogenetic tree constructed from the alignment displayed in (E, F and $G$ ). The Gen Bank accession numbers of sequences used for the alignments are: Ls-IAP2 AAQ11129.1, Ls-IAP3 AAQ11158.1, B. mori IAP (Bm-IAP) AF28107, Spodoptera frugiperda IAP (SfIAP) AF186378, Trichoplusia ni IAP (Tn-IAP) AF195528, Orgyia pseudotsugata nucleopolyhedrosis IAP (Op-IAP) P41437 and Cydia pomonella granulovirus IAP (Cp-IAP) P41436.

or transfected with pIZ-IAP2 was degraded into fragments, showing typical "DNA ladder" configuration (Fig. 5 lane 1 and lane 2).

Ls-IAP3 and Ls-p49 rescued the replication of AcMNPV $\Delta$ p35/pol ${ }^{+}$in $\mathbf{S F 9}$ cells. pIZ-IAP2, pIZ-IAP3 or pIZ-p49 was cotransfected into SF9 cells with AcMNPV $\Delta p 35 /$ pol ${ }^{+}$DNA. 3-4 days after transfection, the cells were examined the presence of polyhedra with light microscopy.

Polyhedra were observed in the cells cotransfected with AcMNPV $\Delta p 35 / \mathrm{pol}^{+}$DNA and pIZ-IAP3 or AcMNPV $\Delta p 35 /$ pol $^{+}$DNA and pIZ-p49 (Fig. 6A4 and A5). No polyhedra were observed in the cells transfected with AcMNPV $\Delta p 35$ / $\mathrm{pol}^{+}$DNA or cotransfected with AcMNPV $\Delta p 35 / \mathrm{pol}^{+}$DNA and pIZ-IAP2 (Fig. 6A2 and A3). To examine polyhedrin in transfected cells, Western-blot was performed using antibody of polyhedrin. Polyhedrin were detected in the cells cotransfected 


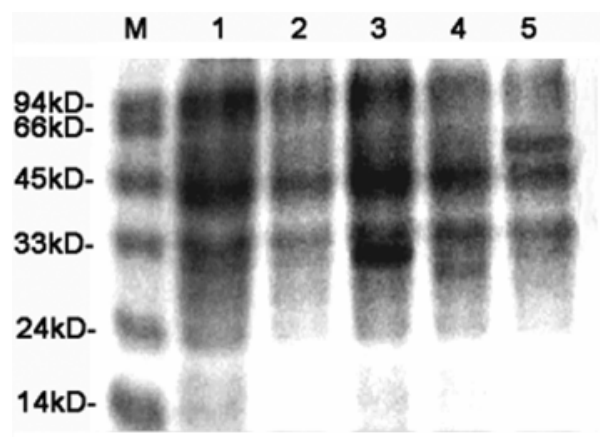

(A)

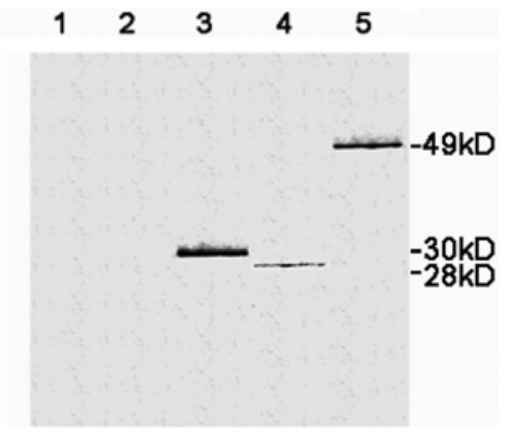

(B)

Fig. 3. SDS-PAGE (A) and Western-blot (B) analysis of Ls-IAP2, Ls-IAP3 and Ls-P49 proteins expressed in SF9 cells. (A) Lane M: protein marker. Lane 1: SF9 cells were untransfected with any plasmid for comparison. Lane 2: SF9 cells only were treated with ActD for $24 \mathrm{~h}$ after culture 12h. Lane 3- Lane 5: $12 \mathrm{~h}$ prior to the addition of ActD, SF9 cells were transfected with pIZ-IAP2 (Lane 3), pIZIAP3 (Lane 4) or pIZ-p49 (Lane 5), respectively and then the transfected cells were treated with ActD for 24 h. (B) Western-blot analysis corresponding to (A).

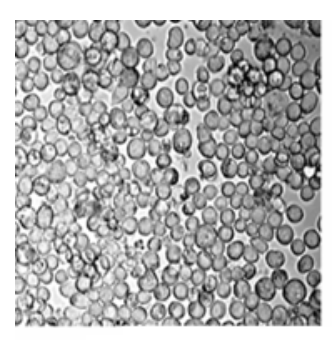

(A)

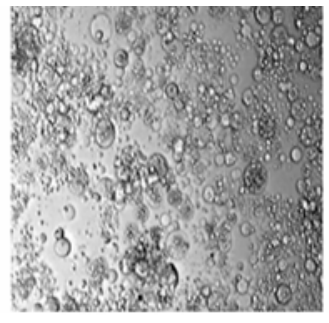

(B)

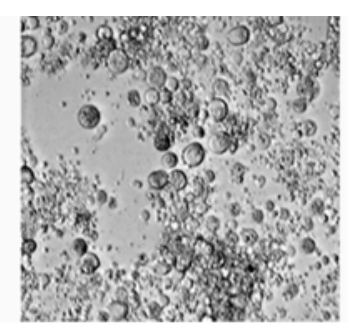

(C)

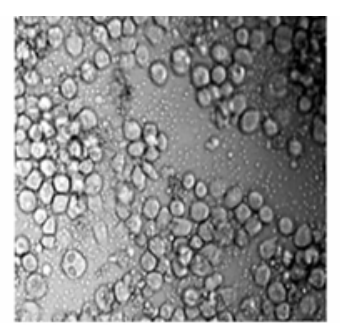

(D)

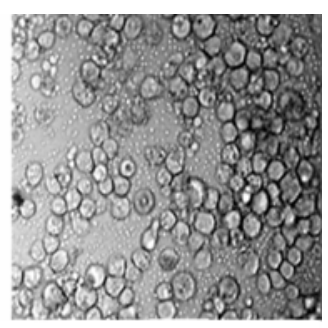

(E)

Fig. 4. Transfection of SF9 cells with pIZ-IAP3 or pIZ-p49 prevents apoptosis induced by actinomycinD (morphology observation of SF9 cells). (A) In the normal SF9 cells, no significant characteristic of apoptosis appeared. (B, C) Cells were mock transfected (B) or transfected with pIZ-IAP2(C) $12 \mathrm{~h}$ prior to adding ActD and then treated with ActD for $24 \mathrm{~h}$, the number of SF9 cells were decreased and the cells shrank. (D, E) Cells were transfected with pIZ-IAP3 (D) or pIZ-p49 (E) 12h prior to adding ActD and then treated with ActD for $24 \mathrm{~h}$, the morphology of SF9 cells looked like the normal cells (A), i.e. no obvious apoptosis characteristics were observed.

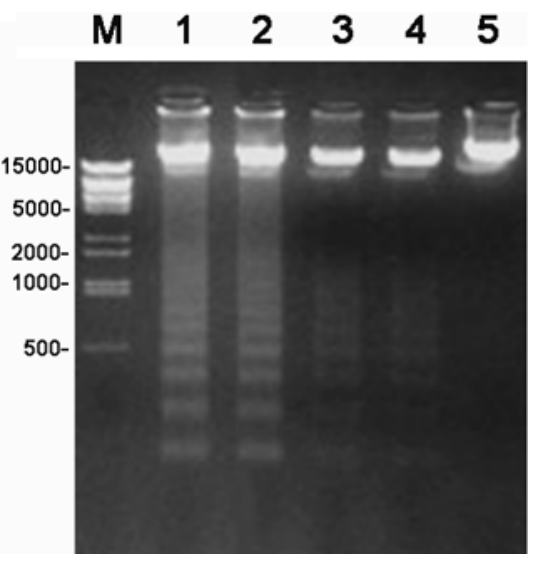

Fig. 5. DNA ladder analysis indicated that Ls-IAP3 and Ls-p49 prevented apoptosis of SF9 cells induced by actinomycin D. Lane 1: Cells were treated with actinomycin D for $24 \mathrm{~h}$. Lane 24: Cells were transfected with pIZ-IAP2 (Lane 2), pIZ-IAP3 (Lane 3) or pIZ-p49 (Lane 4) $12 \mathrm{~h}$ prior to the addition of ActD and then treated with ActD for $24 \mathrm{~h}$. Lane 5: SF9 cells. Lane M: DNA marker. with AcMNPV $\Delta p 35 / \mathrm{pol}^{+}$DNA and Ls-iap3 or AcMNPV $\Delta p 35 /$ pol $^{+}$DNA and Ls-p49 (Fig. 6B4 and B5), but were not in the cells transfected with AcMNPV $\Delta p 35 /$ pol $^{+}$DNA or cotransfected with AcMNPV $\Delta p 35 /$ pol $^{+}$DNA and pIZ-IAP2 (Fig. 6B2 and B3). Such results demonstrate that the expression of Ls-IAP3 and Ls-P49 could rescue the AcMNPV $\Delta p 35 / \mathrm{pol}^{+}$replication in SF9 cells.

The effect of Ls-IAP3 or Ls-P49 protein that could inhibit apoptosis induced by transfection of AcMNPV $\Delta p 35 / \mathrm{pol}^{+}$was also proved by flow cytometry after the cells stained with PI (Fig. 7). As measured by flow cytometry, the apoptosis in group of normal cells is only $0.42 \%$ (Go peak in Fig. 7A), while apoptosis cells (stained by PI) increased to $27.4 \%$ (Fig. 7B) when AcMNPV $\Delta p 35 / \mathrm{pol}^{+}$genome DNA was transfected. When equal amount of AcMNPV $\Delta p 35 / \mathrm{pol}^{+}$genome DNA was cotransfected with plasmid pIZ-IAP3 or pIZ-p49, respectively, the apoptosis decreased to $11.3 \%$ and $13.9 \%$, respectively (Fig. 7C, D), which indicated that Ls-IAP3 and Ls-P49 could partially inhibit the apoptosis caused by AcMNPV $\Delta p 35 / \mathrm{pol}^{+}$ genome in transient experiments. 
(B)

$\begin{array}{lllll}1 & 2 & 3 & 4 & 5\end{array}$

(A)

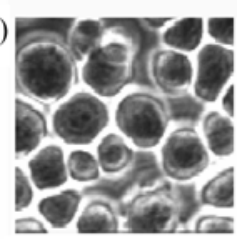

1

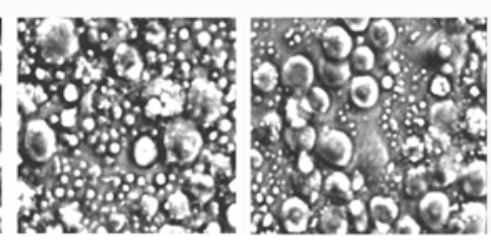

3
- blot: polyhedra

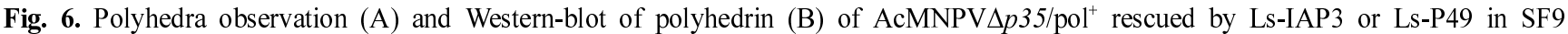
cells. 1: SF9 cells $72 \mathrm{~h}$ after mock transfected. 2: SF9 cells $72 \mathrm{~h}$ after transfected with AcMNPV $\Delta p 35 / \mathrm{pol}^{+}$viral DNA. 3-5: SF9 cells $72 \mathrm{~h}$ after cotransfected with AcMNPV $\Delta p 35 / \mathrm{pol}^{+}$viral DNA and pIZ-IAP2 (3), AcMNPV $\Delta p 35 / \mathrm{pol}^{+}$viral DNA and pIZ-IAP3 (4) or AcMNPV $\Delta p 35 /$ pol $^{+}$viral DNA and pIZ-p49 (5). (B) Western blot of polyhedrin corresponding to A.

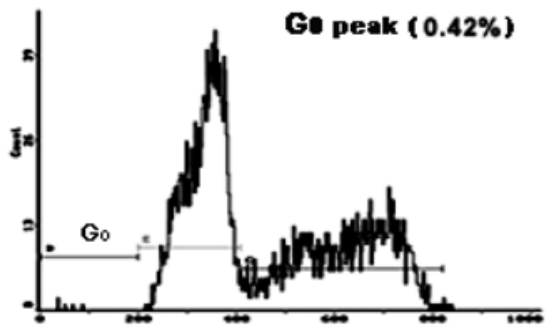

(A)

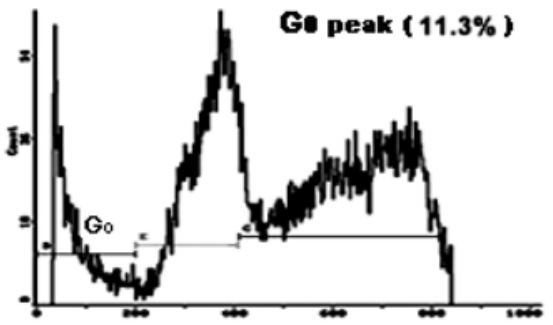

(C)

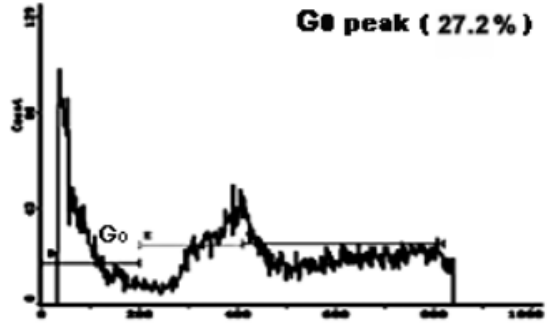

(B)

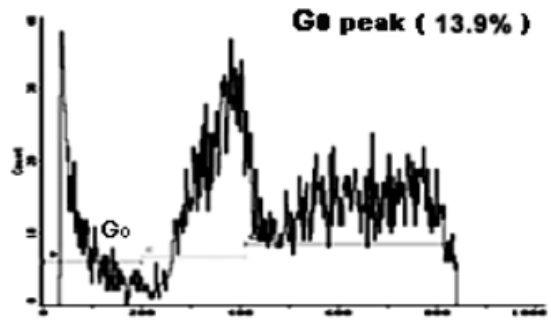

(D)

Fig. 7. The apoptosis assay by flow cytometry after transfection with AcMNPV $\Delta p 35 / \mathrm{pol}^{+}$. (A) No significant apoptosis peak in normal SF9 cells $(0.42 \%)$. (B) $24 \mathrm{~h}$ after transfected with AcMNPV $\Delta p 35 / \mathrm{pol}^{+}$, the apoptosis peak showed that $27.4 \%$ of primary cells undergone apoptosis. (C, D) $24 \mathrm{~h}$ after cotransfected with AcMNPV $\Delta p 35 / \mathrm{pol}^{+}$DNA and pIZ-IAP3 (C) or AcMNPV $\Delta p 35 / \mathrm{pol}^{+}$DNA and pIZ-p49 (D), the ratio of apoptosis cells was $11.3 \%$ (C) or $13.9 \%$ (D), respectively.

\section{Discussion}

Baculoviruses possess two types of genes with anti-apoptotic activity, iap and p35 that can suppress apoptosis induced by virus infection or by diverse stimuli in vertebrates or invertebrates (Pei et al., 2002). The Baculovirus p35 gene have been identified in very few baculoviruses (Clen et al., 1994), while the iap gene have been identified in all baculoviruses studied to date (Crook et al., 1993).

p49 gene, another member of the famous p35 family, was first identified in the Spodoptera littoralis nucleopolyhedrovirus (SINPV) (Du et al., 1999). p49 was only possessed by groupII NPV like SINPV and LsMNPV (Du et al., 1999). The P49 from LsMNPV also contained a reactive site loop (RSL), while had different predicted cleavage site $\left(\mathrm{KKLD}^{74} \downarrow_{\mathrm{G}}\right)$ from S1-p49 (TVID $\left.{ }^{94} \downarrow G\right)$.
Two iap homologues of LsMNPV were designated as LsIAP2 and Ls-IAP3 because of their amino acid sequence homology with other IAP2 and IAP3 that were identified previously in HycuNPV (Ikeda et al., 2004), CpGV (Luque et al., 2001), OpMNPV (Means et al., 2003) and EppoMNPV (Maguire et al., 2000). Hycu-IAP1, -IAP2 and -IAP3 showed the highest homology with corresponding OpMNPV IAPs, exhibiting $81 \%, 71 \%$ and $62 \%$ amino acid sequence identities with Op-IAP1, -IAP2 and -IAP3, respectively (Ikeda et al., 2004), but Ls-IAP2, -IAP3 exhibits $44 \%$ and $60 \%$ amino acid sequence identities with Op-IAP2, -IAP3, respectively. So the identity of IAPs between LsMNPV and OpMNPV was not higher than that of HycuNPV and OpMNPV.

The BIR and RING domains of B.mori IAP (Bm-IAP) are required to block apoptosis induced by AcMNPV $\Delta p 35 / \mathrm{pol}^{+}$ (Huang et al., 2001). Like other baculovirus IAPs, Ls-IAP2 
and IAP3 possess one and two BIR domains in the N-terminal region, respectively, and both IAPs contain a single RING finger motif in the C-terminal region. We tested anti-apoptosis ability of Ls-IAP2, Ls-IAP3 and Ls-p49 using the traditional marker rescue.

Actinomycin D and AcMNPV $\Delta p 35 / \mathrm{pol}^{+}$was used to establish the apoptosis system in SF9 cells. Actinomycin D can suppress cell protein synthesis; induce many kinds of cell to apoptosis (Maguire et al., 2000). SF9 cells infected with AcMNPV $\Delta p 35 /$ pol $^{+}$could induce apoptosis (Chejanovsky and Gershburg, 1995). Our results from transient expression assays in SF9 cells revealed that both Ls-IAP3 and Ls-p49 could block apoptosis of SF9 cells induced by ActD treatment or p35-deficient AcMNPV transfection, while Ls-IAP2 exhibited no detectable anti-apoptotic activity. The result showed that Ls-iap3 and Ls- $p 49$ are two new anti-apoptotic gene homologues that has not been identified before.

Further works should be done to confirm whether the LsIAP3 and Ls-P49 proteins are essential for LsMNPV replication or not. We will attempt to isolate various Ls-IAP3 and Ls-p49 deficient LsMNPV mutants to determine their functional domains, and also investigate the interaction of LsIAP3 and Ls-p49 with insect and human effector caspase. The findings suggest that more anti-apoptosis genes may exist in virus and probably in the animal kingdom.

Acknowledgments This research was supported by grants from the National 973 Project of China (2003CB114002) and from the National Nature Science Foundation of China (30570082).

\section{References}

Birnbaum, M. J., Clem, R. J. and Miller, L. K. (1994) An apoptosis-inhibiting gene from a nuclear polyhedrosis virus encoding a polypeptide with Cys/His sequence motif. J. Virol. 68, 2521-2528.

Chejanovsky, N. and Gershburg, E. (1995) The wild-type autographa californica nuclear polyhedrosis induces apoptosis of spodoptera littoralis cells. Virology 209, 519-525.

Clem, R. J. and Miller, L. K. (1994) Control of programmed cell death by the baculovirus genes P35 and IAP. Mol. Cell. Biol. 14, 5212-5222.

Clem, R. J. (2001) Baculoviruses and apoptosis the good, the bad, and the ugly. Cell Death Differ. 8, 137-143.

Crood, N. E., Jams, J. D., Smith, I. R. L. and Winstanley, D. (1997) Comprehensive; physical map of the Cydia pomonella granulovirus genome and sequence analysis of the granulin gene region. J. Gen. Virol. 78, 974.

Crook, N. E., Clem, R. J. and Miller, L. K. (1993) An apoptosisinhibiting baculovirus gene with a zinc finger-like motif. $J$. Virol. 67, 2168-2174.

Dai, X., Shi, X., Pang, Y. and Su, D (1999) Prevention of baculovirus-induced apoptosis of BT1-Tn-5B1-K(His) cells by the P35 gene of Tricholusia ni multicapsid nucleopolyhedrovirus. J. Gen. Viro. 80, 1841-1845.

Day, C. L. (1999) Solution structure of a baculoviral inhibitor of apoptosis (IAP) repeat. Nat. Struct. Biol. 6, 648-651.

Du, Q., Lehavi, D., Faktor, O., Qi, Y. and Chejanovsky, N. (1999) Isolation of an Apoptosis Suppressor Gene of the Spodoptera Littoralis Nucleopolyhedro virus. J. Virology 73, 1278-1285.

Friesen, P. D. and Miller, L. K. (1987) Divergent transcription of early 35 and 94-kilo Dalton protein genes encoded by the HindIII $\mathrm{K}$ genome fragment of the baculovirus Autographa californica nuclear poly hedrosisvirus. J. Virol. 61, 2264-2272.

Hozak, R. R., Manji, G. A. and Friesen, P. (2000) The BIR motif mediate dominant interference and oligomerization of inhibitor of apoptosis Op-IAP. Mol. Biol. Cell. 20, 1877-1885.

Huang, Q., Deveraux, Q. L., Maeda, S., Salvesen, G. S. Stennjcke, H. R. Hammock, B. D. and Reed, J. C. (2000) Evolutionary conservation of apoptosis mechanism: Lepidopteran and baculoviral inhibitor of apoptosis proteins are inhibitors of mammalian caspase-9. Proc. Natl. Acad. Sci. USA 97, 14271432.

Huang, Q., Deveraux, Q. L., Maeda, S., Stennicke, H. R. Hammock, B. D. and Reed, J. C. (2001) Cloning and characterization of an inhibitor of apoptosis protein (IAP) from Bombyx mori. Biochim. Biophys. Acta. 1499, 191-198.

Ikeda, M., Yanagimoto, K. and Kobayashi, M (2004) Identification and functional analysis of Hyphantria cunea nucleopolyhedrovirus IAP genes. Virology 321, 359-371.

Kamita, S. G., Majima, K. and Maeda, S. (1993) Identification and characterization of the $P 35$ gene of Bombyx mori nuclear polyhedrosis virus that prevents virus-induced apoptosis. $J$. Virol. 62, 455-463.

Luque, T., Finch, R., Crook, D., OReilly, R. and Winstanley, D. (2001) The complete sequence of the Cydia pomonella granulovirus genome. J. Gen. Virol. 82, 2531-2547.

Lin, J., Zhang, C. and Suzuki, S. (2005) UZUKI, Anti-apoptosis ene of the Bcl-2 family from marine birnavirus ihibiting apoptosis of insect cells infected with baculovirus. Virus Genes 31, 185-193.

Maguire, T., Harrison, P., Hyink, O., Kalmakoff, J. and Ward, V. K. (2000) The inhibitors of apoptosis of Epiphyas postvittama nucleopolyhedrovirus, J. Gen. Virol. 81, 2803-2811.

Means, J. C., Muro, I. and Clem, R. J. (2003) Silencing of the baculovirus Op-IAP3 gene by RNA interference reveals that it is required for prevention of apoptosis during Orgyia pseudotsugata $\mathrm{M}$ nucleopolyhedrovirus infection of $\mathrm{Ld} 652 \mathrm{Y}$ cells. J. Virol. 77, 4481-4488.

Pei, Z., Reske, G., Huang, Q., Hammock, B. D., Qi, Y. and Chejanovsky, N. (2002) Characterization of the apoptosis suppressor protein $p 49$ from the Spodoptera littoralis nucleopolyhedrovirus. J. Biol. Chem. 277, 48677-48684.

Rost, B., Yachdav, G. and Liu, J. (2003) The predict protein server. Nucleic Acids Res. 32, 321-326.

Seshagiri, C. S., Bucic, D., Lee, J. and Dixit, V. M. (1999) Baculovirus based genetic screen for antiapoptotic genes identifies a novel IAP. J. Biol. Chem. 274, 36764-36773.

Vilaplana, L. I. and Reilly, D. R. O. (2003) Functional interaction between Cydia pomonella granulovirus IAP proteins. Virus Res. 92, 107-111.

Wang, Y., Qi, Y., Zhu, Y., Li, Z. and Yang, Y. (2003) Functional study on baculovirus anti-apoptosis genes. Mol. Cell. Biochem. 252, 103-107.

Wrght, C. W., Meaus, J. C., Penabaz, T. and Chem, R. J. (2005) The baculovirus anti-apoptotic protein Op-IAP does not inhibit Drosophila caspases or apoptosis in Drosophila S2 cells and instead sensitizes S2 cells to virus-induced apoptosis. Virology $335,61-71$. 